\title{
Stomach- and Site-Selective Delivery of 5-Fluorouracil Following Its Application on the Gastric Serosal Surface in Rats
}

\author{
Junzo Nakamura, ${ }^{a}$ Kazuko Kobayashi, ${ }^{a}$ Shintaro Fumoto, $,{ }^{*}, a$ Junya Nishi, ${ }^{a}$ Takahiro Mukai, ${ }^{a}$ \\ Mikiro NAKashima, ${ }^{b}$ Hitoshi SASAKI,${ }^{b}$ and Koyo NishidA ${ }^{a}$ \\ ${ }^{a}$ Graduate School of Biomedical Sciences, Nagasaki University; 1-14 Bunkyo-machi, Nagasaki 852-8521, Japan: and \\ ${ }^{b}$ Department of Hospital Pharmacy, Nagasaki University Hospital of Medicine and Dentistry; 1-7-1 Sakamoto, Nagasaki \\ 852-8501, Japan. Received December 8, 2004; accepted February 23, 2005
}

The present study was undertaken to elucidate the stomach- and site-selective delivery of 5-fluorouracil (5-FU) following its application on the gastric serosal surface in rats. An experimental system utilizing a cylindrical diffusion cell attached to the gastric serosal surface was established. To evaluate the gastric distribution of 5FU, the stomach was separated into the site under the diffusion cell (site 1) and the site not under the diffusion cell (site 2). Furthermore, the mucosal side at site 1 was separated from the serosal side. After intravenous and oral administration of 5-FU, the 5-FU concentrations at sites 1 and 2 until 240 min were similar. After gastric serosal surface application of 5-FU, however, the concentration of 5-FU at site 1 until 240 min was approximately 10-fold higher than that at site 2, and was sustained. Furthermore, the 5-FU concentration on the mucosal side at site 1 and the serosal side at site 1 were comparable after gastric serosal surface application. The blood concentration of 5-FU was low $(<4.4 \mu \mathrm{g} / \mathrm{ml})$ until $240 \mathrm{~min}$ after gastric serosal surface application. The maximum blood concentration of 5-FU after gastric serosal surface application was significantly lower than after intravenous administration. Thus, the stomach- and site-selective delivery system following application on the gastric serosal surface could be applied with anticancer drugs for the treatment of gastric cancer.

Key words gastric cancer; chemotherapy; stomach targeting; drug delivery system; 5-fluorouracil; rat

Advanced gastric cancer with serosal invasion is usually unresectable, so anticancer drugs are infused intravenously as a palliative treatment; however, patients with unresectable gastric cancer die within a few months after exploration, with or without palliative surgery. 5-Fluorouracil (5-FU) is the cornerstone chemotherapy regimen for gastric cancer, having mild to moderate toxicity and response rate. ${ }^{1)}$ However, the activity of single agents, including 5-FU, against gastric cancer is approximately $20 \%$ or less. $\left.{ }^{2}\right)$ To improve the therapeutic effect of anticancer drugs on gastric cancer, intraarterial administration has been studied. ${ }^{3,4)}$ When the anticancer drugs are administered via the vasculature route, however, they are distributed through the whole body via the blood stream, leading to inadequate delivery to target sites in the stomach, as well as potential toxicity in other organs. These circumstances underscore the need for novel and more effective strategies for the treatment of gastric cancer.

Thus, stomach- and site-selective drug delivery is a very important strategy for the optimization of chemotherapy in terms of efficacy and safety. We originally elucidated that phenol red, bromphenol blue, and bromosulphonphthalein as model drugs are adequately absorbed from the gastric serosal surface and accumulate site-selectively in the stomach in rats. ${ }^{5,6)}$ Although the gastric serosal surface application of anticancer drugs holds promise for site-selective delivery in the stomach, little is known about the gastric and systemic distribution characteristics of anticancer drugs following application on the gastric serosal surface. In the present study, we examined the detailed absorption and distribution characteristics of 5-FU following application on the gastric serosal surface for the site-selective delivery of 5-FU in the stomach in rats.

\section{MATERIALS AND METHODS}

Chemicals 5-FU was purchased from Nacalai Tesque, Inc. (Kyoto, Japan). All other chemicals were of reagent grade.

Absorption and Distribution Experiments All experiments in the present study were carried out in accordance with the Guidelines for Animal Experimentation of Nagasaki University. Male Wistar rats $(250-270 \mathrm{~g})$ were anesthetized with sodium pentobarbital $(50 \mathrm{mg} / \mathrm{kg}$ i.p.). After the peritoneum was dissected about $5 \mathrm{~cm}$, a cylindrical diffusion cell (i.d. $9 \mathrm{~mm}$, effective area $0.64 \mathrm{~cm}^{2}$ ) was attached to the gastric serosal surface with surgical adhesive (Aron Alpha A, Sankyo Co. Ltd., Tokyo, Japan), and 5-FU (0.2, 2, and $10 \mathrm{mg} / \mathrm{ml} \times 0.5 \mathrm{ml}$, isotonic phosphate-buffered saline, $\mathrm{pH}$ 7.4) was added directly to the cylindrical diffusion cell (Chart 1). The body temperature of the rats was maintained at $37^{\circ} \mathrm{C}$ with a heat lamp during the experiment. As a control experiment, 5-FU $(10 \mathrm{mg} / \mathrm{ml} \times 0.5 \mathrm{ml}$, isotonic phosphatebuffered saline, $\mathrm{pH}$ 7.4) was administered intravenously using a syringe with a needle $\left(26 \mathrm{G} \times 1 / 2^{\prime \prime}\right)$ and orally by gastric intubation. After application on the gastric serosal surface, the solution in the diffusion cell and the blood was sampled at appropriate times. Subsequently, the rats were sacrificed, and the stomach, small intestine, liver, kidney, and heart were removed at predetermined times. To evaluate the gastric distribution of 5-FU, the stomach was separated into the site under the diffusion cell (site 1) and the site not under the diffusion cell (site 2); furthermore, the mucosal side at site 1 was separated from the serosal side by scraping using a spatula (Chart 2). The tissues were weighed, then site 1, the mucosal side at site 1 , the serosal side at site 1 , and other tissues including site 2 were homogenized in 19, 14, 14, and 2fold volumes of isotonic phosphate-buffered saline, $\mathrm{pH} 7.4$, respectively. 


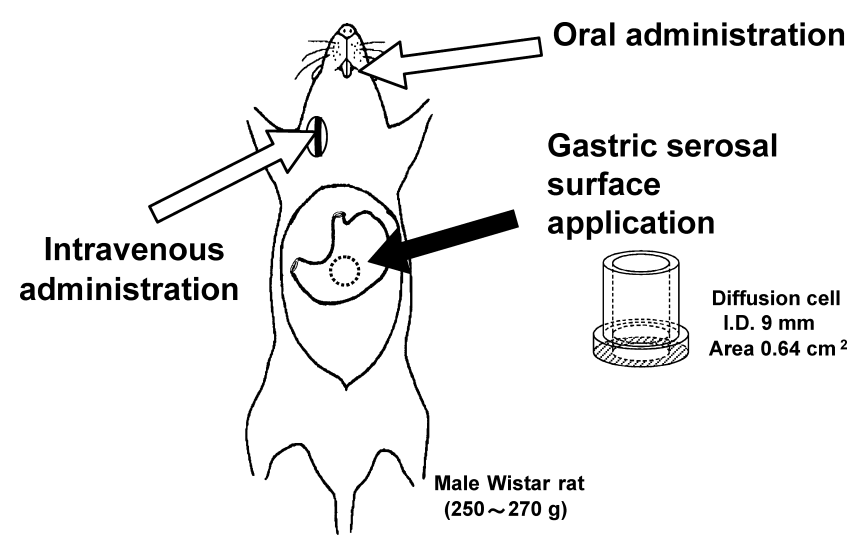

Chart 1. Experimental Procedures for Drug Application in Rats

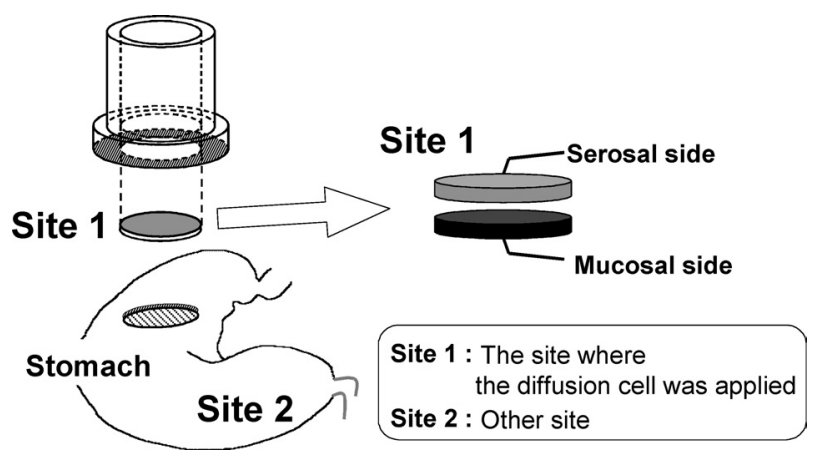

Chart 2. Division of Gastric Site 1 to the Serosal Side and Mucosal Side

5-FU Assay Procedures in Blood and Tissues The concentration of 5-FU in each tissue homogenate or blood sample was determined by the reported method with slight modification. ${ }^{7)}$ Briefly, the tissue homogenates $(300 \mu \mathrm{l})$ and blood samples $(300 \mu \mathrm{l})$ were added to a solution of 5-bromouracil $(20 \mu \mathrm{g} / \mathrm{ml}, 150 \mu \mathrm{l})$ dissolved in isotonic phosphate buffer (pH 7.4) as an internal standard, $1 \mathrm{~m}$ sodium acetate buffer ( $\mathrm{pH} 4.8,100 \mu \mathrm{l}$ ), and $20 \%$ anhydrous sodium sulfate solution $(500 \mu \mathrm{l})$. The mixtures were shaken with ethyl acetate $(4 \mathrm{ml})$ for $10 \mathrm{~min}$ and centrifuged at $900 \times \boldsymbol{g}$ for $10 \mathrm{~min}$. The organic layers $(3 \mathrm{ml})$ were collected. Then, ethyl acetate $(4 \mathrm{ml})$ was added to the residue and the mixtures were shaken for $10 \mathrm{~min}$, and thereafter centrifuged at $900 \times \boldsymbol{g}$ for $10 \mathrm{~min}$. The organic layers $(4 \mathrm{ml})$ were collected and the mixed organic layers $(7 \mathrm{ml})$ were evaporated. The extraction residues were dissolved in $500 \mu \mathrm{l}$ of distilled water and were washed twice with $1.0 \mathrm{ml}$ of hexane. Samples $(100 \mu \mathrm{l})$ were injected onto the HPLC column. The recovery rates for the extraction of 5FU from blood and each tissue homogenate were approximately $75 \%$. The detection limits of 5 -FU in the blood and each tissue homogenate were $0.2 \mu \mathrm{g} / \mathrm{ml}$. An HPLC system (LC-6A, Shimadzu Co., Ltd., Kyoto, Japan) with a variablewavelength UV detector (SPD-10A, Shimadzu) was used in reverse-phase mode. The detector wavelength, flow rate, and column temperature were set at $266 \mathrm{~nm}, 0.5 \mathrm{ml} / \mathrm{min}$, and $25^{\circ} \mathrm{C}$, respectively. The mobile phase consisted of $10 \mathrm{~mm}$ sodium acetate buffer ( $\mathrm{pH} 4.0$ ). The stationary phase used was a Cosmosil $5 \mathrm{C}_{18}$-MS-II packed column $(150 \mathrm{~mm}$ length $\times 4.6 \mathrm{~mm}$ i.d. connected with $150 \mathrm{~mm}$ length $\times 4.6 \mathrm{~mm}$ i.d., Nacalai Tesque, Inc.).

Elimination of 5-FU from the Stomach after the Re-



Fig. 1. Semi-log Plot of the Remaining Amount of 5-FU in the Diffusion Cell until $240 \mathrm{~min}$ after the Gastric Serosal Surface Application of 5-FU at Doses of $0.1(\bigcirc), 1.0(\bigcirc)$, and $5.0(\triangle) \mathrm{mg}$ in Rats, Respectively

Each value represents the mean \pm S.E. of at least four experiments.

moval of 5-FU in the Diffusion Cell The rats were anesthetized with sodium pentobarbital. After the peritoneum was cut open, the cylindrical diffusion cell was attached to the gastric serosal surface, and 5 -FU $(10 \mathrm{mg} / \mathrm{ml} \times 0.5 \mathrm{ml}$, isotonic phosphate-buffered saline, $\mathrm{pH}$ 7.4) was added directly to the cylindrical diffusion cell. After $120 \mathrm{~min}, 5$-FU was removed from the cylindrical diffusion cell and the gastric serosal surface in the diffusion cell was washed by saline $(0.5 \mathrm{ml}) 3$ times. At 15 and $30 \mathrm{~min}$ after the removal of 5-FU, the rats were sacrificed, and the stomach was removed. The stomach was separated into the site under the diffusion cell (site 1) and the site not under the diffusion cell (site 2). The tissues were weighed and homogenized in isotonic phosphatebuffered saline, $\mathrm{pH}$ 7.4.

\section{RESULTS}

Absorption of 5-FU from the Gastric Serosal Surface We established an experimental system utilizing a cylindrical diffusion cell attached to the gastric serosal surface in rats (Chart 1). This system enables us to examine the drug absorption from the gastric serosal surface without interference by absorption from the other organs and sites. ${ }^{5}$ We examined the absorption characteristics of 5-FU following its application on the gastric serosal surface until $240 \mathrm{~min}$ at doses of $0.1,1.0$, and $5.0 \mathrm{mg}$ in rats (Fig. 1). After the gastric serosal surface application of 5-FU, 5-FU was absorbed approximately $60 \%$ in $240 \mathrm{~min}$ at each dose. A semi-log plot of the remaining amount of 5-FU in the diffusion cell gave a straight line, indicating that the absorption of 5-FU from the gastric serosal surface proceeds via a first-order process. The absorption rate constant $K_{\mathrm{a}}$ of 5-FU from the gastric serosal surface at doses of $0.1,1.0$, and $5.0 \mathrm{mg}$ was calculated to be $0.00413 \pm 0.00033,0.00435 \pm 0.00022$, and $0.00388 \pm 0.00029 \mathrm{~min}^{-1}$, respectively, and there was no significant difference (one-way analysis of variance). From these results, it is suggested that the absorption of 5-FU from the gastric serosal surface is explained mostly by passive diffusion.

Gastric Distribution of 5-FU Figure 2 shows the gastric concentration profiles of 5-FU until $240 \mathrm{~min}$ after intravenous administration (A), oral administration (B), and gastric serosal surface application (C) of 5-FU. After intravenous and oral administration of 5-FU, the concentrations of 

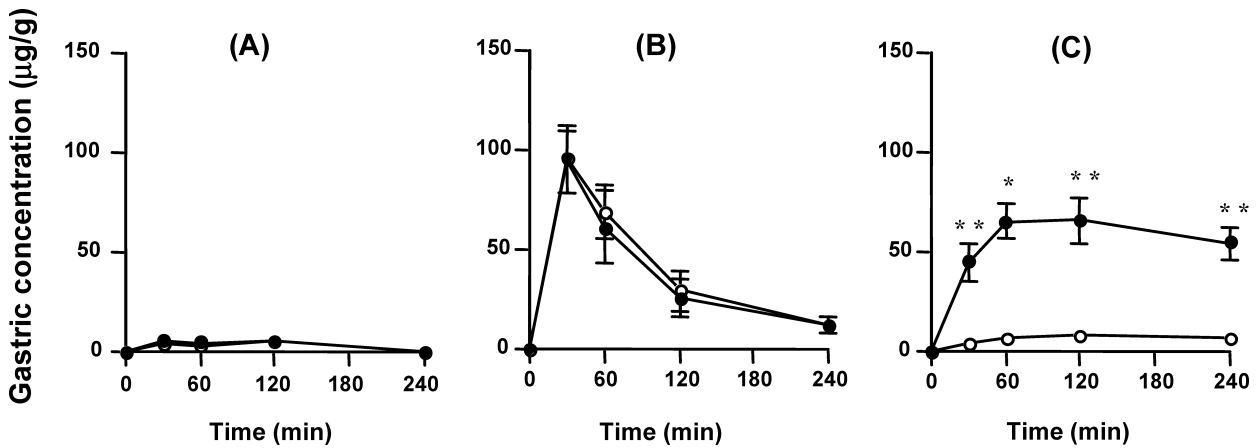

Fig. 2. Gastric Concentration of 5-FU until 240 min after the Intravenous Administration (A), Oral Administration (B), and Gastric Serosal Surface Application (C) of 5-FU at a Dose of $5 \mathrm{mg}$ in Rats

5-FU was determined at site $1(\bullet)$ and site $2(\bigcirc)$. Statistical comparisons were performed using the Student's $t$ test (paired) $(* p<0.05$ and $* * p<0.01$, significantly different from at site 2). Each value represents the mean \pm S.E. of at least four experiments.
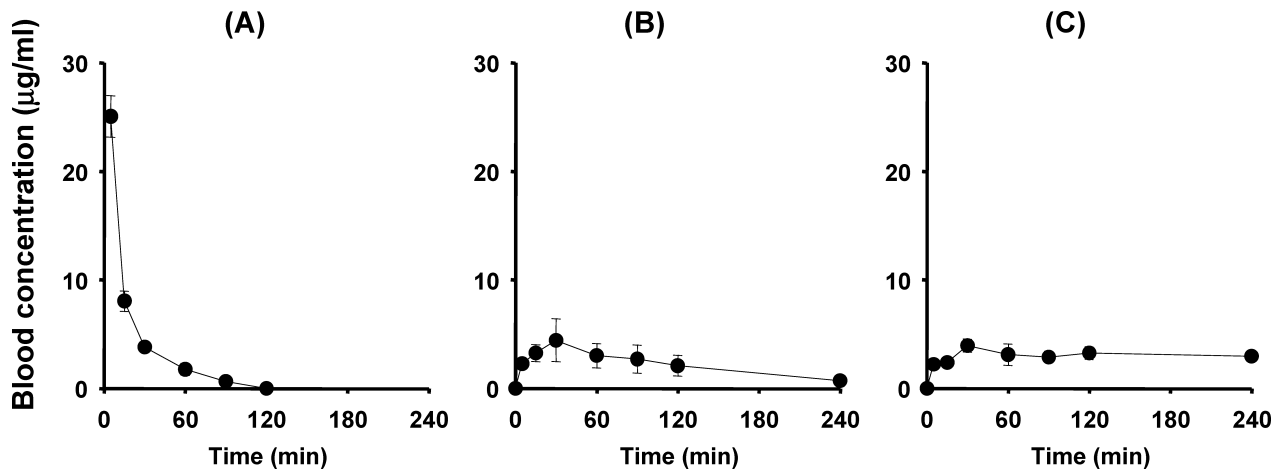

Fig. 3. Blood Concentration of 5-FU until 240 min after the Intravenous Administration (A), Oral Administration (B), and Gastric Serosal Surface Application (C) of 5-FU at a Dose of $5 \mathrm{mg}$ in Rats

Each value represents the mean \pm S.E. of at least four experiments.

5-FU at site 1 and site 2 were similar (Figs. 2A, B). After the gastric serosal surface application of 5-FU, on the other hand, the concentration of 5-FU at site 1 was approximately 10 fold higher than at site 2, and was sustained for $240 \mathrm{~min}$ (Fig. 2C). The 5-FU concentrations on the mucosal and serosal side in the stomach following intravenous and oral administration were not determined.

Systemic Distribution of 5-FU The blood concentration profiles of 5-FU after intravenous administration (A), oral administration (B), and gastric serosal surface application (C) were evaluated (Fig. 3). After oral administration and gastric serosal surface application of 5-FU, a low blood concentration $(<4.4 \mu \mathrm{g} / \mathrm{ml})$ was seen until $240 \mathrm{~min}$. The maximum blood concentration after the oral administration and gastric serosal surface application was significantly lower than after intravenous administration.

Figure 4 shows the concentration profiles of 5-FU in the stomach (site 1 and site 2), small intestine, liver, kidney, and heart until $240 \mathrm{~min}$ after intravenous administration (A), oral administration (B), and gastric serosal surface application (C) of 5-FU. The gastric concentrations of 5-FU after intravenous and oral administration are shown as the whole stomach because the 5-FU concentrations at site 1 and site 2 were similar (Fig. 2). After intravenous administration of 5-FU, 5FU was mainly distributed in the kidney, and this gastric concentration of 5-FU was much lower than that of the other administration routes (Fig. 4A). After oral administration of 5FU, 5-FU was mainly distributed in the stomach, but was rapidly eliminated from the stomach (Fig. 4B). In contrast, after gastric serosal surface application of 5-FU, the concentration of 5-FU at site 1 was 10 -fold higher than the concentration at site 2 and in other tissues (Fig. 4C).

Elimination of 5-FU from the Stomach after the Removal of 5-FU in the Diffusion Cell Figure 5 shows the remaining 5-FU concentration until $30 \mathrm{~min}$ at site 1 and site 2 after the removal of 5-FU after gastric serosal surface application for $120 \mathrm{~min}$. The rapid elimination of 5-FU from site 1 was observed.

Gastric Distribution at the Serosal and Mucosal Sides of Site 1 Figure 6 shows the gastric distribution of 5-FU until $240 \mathrm{~min}$ after the gastric serosal surface application of 5-FU. To evaluate the longitudinal distribution of 5-FU at site 1 , the mucosal side was separated from the serosal side (Chart 2). After the gastric serosal surface application of 5FU, 5-FU concentrations on the mucosal and serosal side at site 1 were from 8 to 27 -fold higher than those at site 2 . Furthermore, the 5-FU concentrations on the mucosal and serosal side at site 1 were comparable.

\section{DISCUSSION}

Gastric cancer still represents one of the most challenging therapeutic problems. Although diagnostic and technological advances have improved perioperative care, gastric cancer remains the leading cause of death from malignant disease. ${ }^{1,8)}$ For effective therapy with medication, it is necessary 

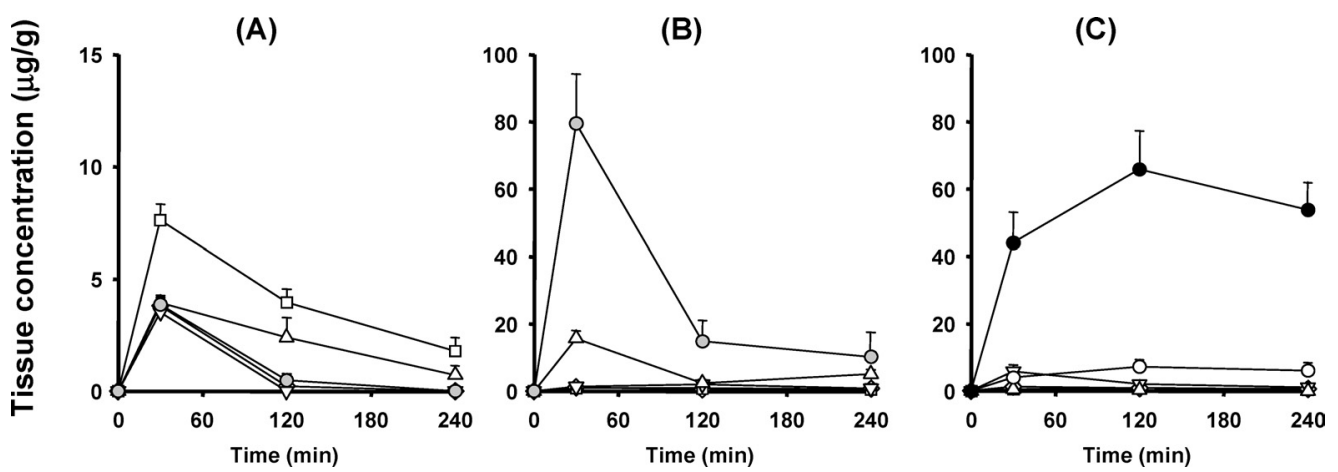

Fig. 4. Tissue Concentration of 5-FU until 240 min after the Intravenous Administration (A), Oral Administration (B), and Gastric Serosal Surface Application (C) of 5-FU at a Dose of $5 \mathrm{mg}$ in Rats

5-FU was determined in the whole stomach $(\bigcirc)$, site $1(\bullet)$, site $2(\bigcirc)$, small intestine $(\triangle)$, liver $(\nabla)$, kidney $(\square)$, and heart $(\diamond)$. Each value represents the mean \pm S.E. of at least three experiments.

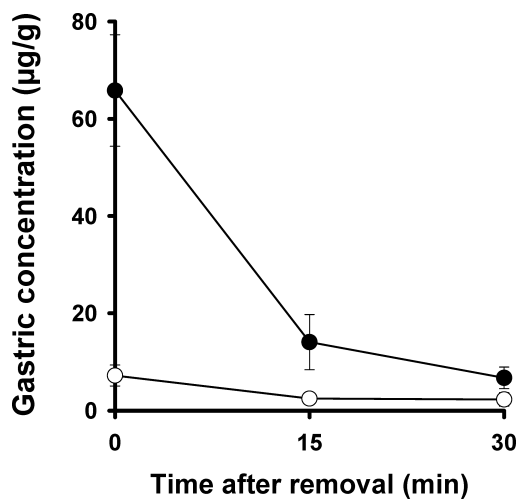

Fig. 5. The Remaining 5-FU until $30 \mathrm{~min}$ in the Stomach after the Gastric Serosal Surface Application of 5-FU at a Dose of $5 \mathrm{mg}$ for $120 \mathrm{~min}$ in Rats

5-FU at site $1(\bigcirc)$ and site $2(O)$ was determined. Each value represents the mean \pm S.E. of at least four experiments.

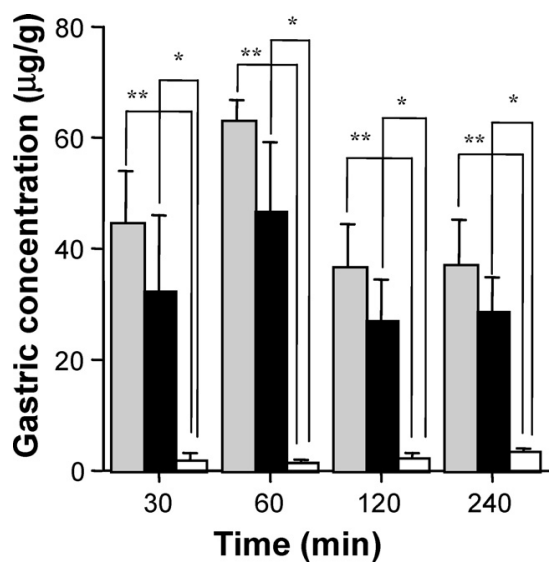

Fig. 6. Gastric Concentration of the Serosal Side at Site $1(\square)$, Mucosal Side at Site $1(\square)$, and Site $2(\square)$ of 5-FU until 240 min after the Gastric Serosal Surface Application of 5-FU at a Dose of $5 \mathrm{mg}$ in Rats

Statistical comparisons were performed using analysis of variance $(* p<0.05$, and $* * p<0.01$, significantly different from at site 2 ). Each value represents the mean \pm S.E of at least three experiments.

to deliver anticancer drugs selectively to their target sites, since most anticancer drugs are associated with both beneficial effects and unfavorable actions. Also, it is important to elucidate the absorption mechanism of an anticancer drug following the intraperitoneal administration in cancer chemotherapy.

Gastric cancer is generated in the gastric mucosal side and then invades the gastric serosal side. ${ }^{9)}$ For effective chemotherapy of gastric cancer, therefore, a promising approach is to deliver the 5-FU to the mucosa following application on the gastric serosal surface. After the gastric serosal surface application of 5-FU, the 5-FU concentration on the mucosal and serosal sides at site 1 were comparable (Fig. 6); consequently, 5-FU was efficiently distributed to the mucosal side from the serosal side prior to distribution to the systemic circulation. These results suggest that the method of gastric serosal surface application of 5-FU could be applied to the chemotherapy of the both intramucosal cancer and intraserosal cancer in the stomach.

The low response rate of anticancer drugs against gastric cancer was suggested to be due to side effects induced by anticancer drugs before they were able to exert a significant anticancer effect; therefore, the side effects of anticancer drugs are important problems to overcome in cancer chemotherapy. ${ }^{10)}$ The common clinical systemic side effects of 5-FU are myelosuppression, stomatitis, nausea, emesis, and diarrhea after intravenous and/or oral administration, ${ }^{11}$ ) and these might be caused by the high blood concentration and non-specific distribution of 5-FU. Accordingly, the blood concentration profiles of 5-FU after intravenous administration, oral administration, and gastric serosal surface application were evaluated as an index of the systemic side effects of 5-FU (Fig. 3). After oral administration and the gastric serosal surface application of 5-FU, a low blood concentration $(<4.4 \mu \mathrm{g} / \mathrm{ml})$ was observed until $240 \mathrm{~min}$. The maximum blood concentration of 5-FU after oral administration and gastric serosal surface application was markedly lower than after intravenous administration. After the gastric serosal surface application of 5-FU, the concentration of 5-FU in other tissues was significantly lower than that of site 1 in the stomach (Fig. 4C). These results suggest that the gastric serosal surface application of 5-FU could diminish the systemic side effects during chemotherapy for gastric cancer, and accordingly, the administration doses of 5-FU could be increased for enhancing the anticancer effects.

The combined uses of various anticancer drugs by intravenous and oral administration were reported to enhance therapeutic effects against gastric cancer. ${ }^{12-14)}$ Therefore, the combination uses of other anticancer drugs with the applica- 
tion of 5-FU on the gastric serosal surface would be expected to enhance the therapeutic effects against gastric cancer.

Although the gastric arterial injection of anticancer drugs was studied to target the delivery of anticancer drugs to the stomach, ${ }^{3,4)}$ it was difficult to remove the injected anticancer drugs while the serious side effects were occurring after administration. In contrast, the 5-FU that accumulated at site 1 after application on the gastric serosal surface was rapidly eliminated from site 1 after removal of the 5-FU in the cylindrical diffusion cell (Fig. 5), suggesting that the interruption of the administration of anticancer drugs would reduce severe side effects immediately.

It has been reported that the intratumoral injection of anticancer drugs could enhance the potency of anticancer drugs by pharmaceutical modifications. ${ }^{15)}$ However, the direct injection of anticancer drugs poses safety concerns because of injury to organs by the needles and the administration volume limits, etc. As shown in Fig. 5, the rapid elimination of 5-FU accumulated at site 1 suggested that even if 5-FU was directly injected into the stomach wall, it would be rapidly eliminated from the injected site. On the other hand, 5-FU was not only site-selectively, but also continuously, delivered to the stomach utilizing the absorption on the gastric serosal surface (Fig. 2C; Fig. 6), suggesting the effectiveness of this administration method.

We previously reported that liver site-selective drug accumulation was enhanced by gradually and continuously instilling a small amount of drug solution on the liver surface in rats. ${ }^{16)}$ Recently, implantable infusion pumps have been developed for the treatment of several diseases, ${ }^{17)}$ and endoscopic and laparoscopic operation techniques have made marked progress. ${ }^{18,19)}$ Furthermore, continuous ambulatory peritoneal dialysis is an extremely popular treatment modality for end stage renal failure; ${ }^{20}$ consequently, the skilled catheter insertion into the intraperitoneal organs should be advanced in the future. It was reported that a collagen fleece coated with fibrin glue could be inserted on the liver and stomach by endoscopic surgery, ${ }^{19)}$ suggesting that proper pharmaceutics with drugs, which could achieve controlled drug release, could be applied to the gastric serosal surface by endoscopic surgery. Taking these findings into consideration, the application of suitable medical skill should make possible the clinical application of 5-FU to the gastric serosal surface.

In summary, we demonstrated the stomach- and site-selective delivery of 5-FU utilizing absorption on the gastric serosal surface in rats. Furthermore, 5-FU applied on the gastric serosal surface was efficiently delivered to the gastric mucosal side. The direct injection of drugs can injure organs with the needle; consequently, such treatment is limited in terms of injecting the drug continually. In contrast, the gastric serosal surface application does not stress the stomach, and therefore continuous administration may be possible to enable the long-term application of 5-FU. Thus, the stomach- and site-selective delivery of 5-FU following application on the gastric serosal surface could be applied for clinical chemotherapy to the gastric cancer. Such information should be useful in the development of a novel administration method for anticancer drugs to targeted sites in the stomach.

Acknowledgments The authors thank Toshiharu Ikeguchi and Daisuke Yamagami for technical assistance. This work was supported in part by a Grant-in-Aid for Scientific Research from the Ministry of Education, Culture, Sports, Science and Technology, Japan, by a Grant-in-Aid from the Uehara Memorial Foundation and by a Grant-in-Aid for Scientific Research from the President of Nagasaki University.

\section{REFERENCES}

1) Vivo R. D., Pignata S., Palaia R., Parisi V., Daniele B., J. Clin. Gastroenterol., 30, 364-371 (2000).

2) Macdonald J. S., Gohmann J. J., Semin. Oncol., 15, $42-49$ (1988).

3) Shchepotin I. B., Chorny V., Hanfelt J., Evans S. R. T., J. Gastrointest. Surg., 3, 426-431 (1999).

4) Iida T., Hirata N., Hirakawa M., Noguchi T., Radiat. Med., 21, 172177 (2003).

5) Nakamura J., Tsurumaru A., Mera K., Mukai T., Nishida K., Sasaki H., Pharm. Pharmacol. Commun., 5, 519-522 (1999).

6) Mukai T., Tsurumaru A., Mera K., Nishida K., Nakamura J., Sasaki H., Sakaeda T., Pharm. Pharmacol. Commun., 5, 609-614 (1999).

7) Kawakami S., Horimoto T., Nishida K., Hirayama R., Mukai T., Nakashima M., Sasaki H., Sakaeda T., Nakamura J., Biol. Pharm. Bull., 25, 928-930 (2002).

8) Sasako M., J. Gastroenterol., 35, 116-120 (2000).

9) Janunger K. G., Hafström L., Nygren P., Glimelius B., Acta Oncol., 40, 309-326 (2001).

10) Shirasaka T., Shimamoto Y., Fukushima M., Cancer Res., 53, 4004 4009 (1993).

11) Yoshida T., Araki E., Iigo M., Fujii T., Yoshino M., Shimada Y., Saito D., Tajiri H., Yamaguchi H., Yoshida S., Yoshino M., Ohkura H., Yoshimori M., Okazaki N., Cancer Chemother. Pharmacol., 26, 352354 (1990).

12) Kelsen D., Atiq O. T., Saltz L., Niedzwiecki D., Ginn D., Chapman D., Heelan R., Lightdale C., Vinciguerra V., Brennan M., J. Clin. Oncol., 10, 541-548 (1992).

13) Shirao K., Shimada Y., Kondo H., Saito D., Yamao T., Ono H., Yokoyama T., Fukuda H., Oka M., Watanabe Y., Ohtsu A., Boku N., Fujii T., Oda Y., Muro K., Yoshida S., J. Clin. Oncol., 15, 921-927 (1997).

14) Cascinu S., Baldelli A. M., Catalano V., Giordani P., Beretta G. D., Silva R. R., Gasparini G., Mari D., Maisano R., Salvagni S., Barni S., Labianca R., Frontini L., Curti C., Catalano G., Br. J. Cancer, 86, 213-217 (2002).

15) Kuang L., Yang D. J., Inoue T., Liu W. C., Wallace S., Wright K. C., Anticancer Drugs, 7, 220-227 (1996).

16) Nakamura J., Yoshida Y., Mera K., Mukai T., Nishida K., Sasaki H., Biol. Pharm. Bull., 22, 713-715 (1999).

17) Hepp K. D., Diabetologia, 37, S108-S111 (1994).

18) Stellato T. A., Surg. Clin. North Am., 72, 997-1002 (1992).

19) Scheyer M., Zimmermann G., Surg. Endosc., 10, 501-503 (1996)

20) Kim C. Y., Kumar A., Sampath L., Sokol K., Modak S., Am. J. Kidney Dis., 39, 165-173 (2002). 